\title{
The Role of the Sciton Profile Nd-Yag Laser in the Reduction of Cutaneous Varicosities, a Pilot Study
}

\author{
Rex Moulton Barrett*, Isabella Kobylinska and Lisa Derita \\ Alameda Hospital, USA
}

*Corresponding author: Rex Moulton Barrett, Alameda Hospital, USA

ARTICLE INFO
Received: 慧July 08, 2019
Published: ${ }^{-1}$ July 22, 2019
Citation: Rex Moulton Barrett, Isabella
Kobylinska, Lisa Derita. The Role of the
Sciton Profile Nd-Yag Laser in the Re-
duction of Cutaneous Varicosities, a Pi-
lot Study. Biomed J Sci \& Tech Res 19(5)-
2019. BJSTR. MS.ID.003365.

\begin{abstract}
\section{Summary}

The current pilot study reports a 19 patient case series to determine the effectiveness of the Sciton Profile Nd- YAG laser in the reduction of cutaneous vessels. Documentation was made of vessel characteristics including color, size, location, percentage reduction and laser settings including pulse width, fluence, numbers of synchronous passes and frequency of applications. We conclude that for vessels less than $4 \mathrm{~mm}$ in diameter, using between 120 350 Joules $/ \mathrm{cm}^{2}, 15-20$ millisecond duration and 1-3 passes, resulted in greater than $50 \%$ clearance without, complication for both red and blue-black vessels.
\end{abstract}

\section{Introduction}

The precise role of the Nd-Yag in the treatment of cutaneous varicosities remains controversial. Treatment choices include sclerotherapy [1,2], laser [3], dual wavelength laser [4], combination sclerotherapy /laser [5,6,7], end-venous laser [8] or radiofrequency [9] and microsurgical or conventional phlebectomy [10]. The vascular literature supports the primary use of sclerotherapy for vessels less than $5 \mathrm{~mm}$ [7]. Some unattractive features of sclerotherapy include the need of the operator to be comfortable using an end-venous injectable. The latter requires a greater degree of dexterity and longer clinical experience than for the use of most lasers in order to achieve a satisfactory result [4].

Laser therapy is also an attractive adjunct or alternative in patients with needle phobia, post-sclerotherapy complications such as neovascularization, otherwise known as matting, the rare person with sclerosant allergy and hemosiderin / brown staining [1]. The latter can be avoided by aspiration of a superficial coagulated vessel, also requiring specific operator skill [2]. Postsclerotherapy complication rates are variable and in part seem dependent on the concentration of a sclerosant used [11]. Variables which may influence overall success of laser therapy include the laser manufacturer's design and calibrations, wavelength, fluence, pulse duration, numbers of consecutive pulses per treatment, pulse interval, pre/concomitant/post cooling, cooling temperature, degree of vascular compression during treatment, application of topical anesthetics, Fitzpatrick skin type, use of post laser compression garments, frequency and interval of retreatments and presence or absence of untreated superficial vein and perforator incompetence [12].

There are at least 6 commercially available wavelengths (532$1064 \mathrm{nms}$ ), 50 laser machines from over 25 manufacturers in the United States today $[13,14]$. The Nd-Yag has several advantages over other laser devises available to treat cutaneous varicosities. The longer $1064 \mathrm{~nm}$ wavelength, provides a relative sparing of the competing chromophore-melanin, including safe usage on Fitzpatrick III-IV skin types. The laser energy is more likely to pass through the epidermis and dermis to be absorbed by oxy and reduced hemoglobin within larger diameter and deeper vessels than for shorter wavelength devices [15]. Important parameters, which must be acknowledged, include a pulse interval in excess of the thermal relaxation time of skin. The thermal relaxation time is dependent the size of the vessel. Vessel coagulation occurs at greater than $62 \mathrm{C}$ and vaporization over 100C [7]. The latter leads to bubble formation within the vessel wall and collapse. The clinical correlation of the latter is termed vessel 'clearing'. A longer pulse width leads to slower heating of the vessel, which in turn avoids vessel rupture [16]. 
Shorter wavelength lasers, while providing greater selectivity for the vascular chromophore, also have a greater propensity towards ablative injury to the vessel wall, which leads to rupture, ecchymosis and potential staining. Superficial varicosities of less than $1 \mathrm{~mm}$ are generally lighter in color and are called spider telangiectasia / veins. Vessels 1-2 $\mathrm{mm}$ in size are termed venectasia and the deeper/darker varicosities of $2-4 \mathrm{~mm}$ diameter are termed reticular vessels [12]. Previous studies using the Nd-Yag to reduce cutaneous vessels have provided a spectrum of recommended parameters from $40-350 \mathrm{~J} / \mathrm{cm}^{2}$ fluence and pulse widths from 1-55 milliseconds (see Table 1). Most authors have reported greater success with reticular vessels than for spider telangiectasia $[4,12]$. Previous authors have noticed that a shorter pulse width (15-30 milliseconds) and greater fluence (120-150 J/ $\left.\mathrm{cm}^{2}\right)$ is required for smaller vessels than for larger vessels: 30-50 milliseconds, 90-120/ $\mathrm{cm}^{2}[12]$.

Table 1: Reported laser parameters from for Nd-YAG reduction of cutaneous veins.

\begin{tabular}{|c|c|c|c|c|c|}
\hline Author Vessel Size(mm) & Spot Size (mm) & Fluence $\mathrm{J} / \mathrm{cm}^{2}$ & Pulse Duration (ms) & Number of passes & Number of passes \\
\hline \multirow{3}{*}{ Weiss \& Weiss, 1999 [17] } & $0.3-0.6$ & - & $80-100$ & 4-Mar & 3 \\
\hline & $0.6-1$ & - & $100-110$ & 10 & 2 \\
\hline & 3-Jan & - & $110-130$ & 7 & 2 \\
\hline Colaiuda, et al. [18] & $<3$ & 6 & $40-150$ & 16-Jan & 3 \\
\hline Wilder, 1999 [19] & 4-Feb & 6 & $145-150$ & 14-Oct & 3 \\
\hline Major, et al. [20] & $0.1-3$ & 2.5 & $70-200$ & $30-0 c t$ & 1 \\
\hline Rothaus, [21] & $1.5-4$ & - & $175-220$ & 50 & - \\
\hline Eremia, et al [15] & $0.3-3$ & 6 & 150 & $25-100$ & 1 \\
\hline \multirow{3}{*}{ Rogachefsky, 2002 [4] } & $0.25-1$ & 7 & $149-150$ & 15 -Oct & 1 \\
\hline & $1.1-2$ & 7 & $100-140$ & $15-40$ & 1 \\
\hline & 4-Feb & 7 & $80-100$ & $40-50$ & 1 \\
\hline Omura et al. [22] & 3-Jan & - & 100 & 50 & - \\
\hline \multirow[t]{2}{*}{ Sackick, 2001 [23] } & $0.2-2$ & 6 & 120 & 7 & 2 \\
\hline & 4-Feb & 6 & 150 & 14 & 1 \\
\hline \multirow[t]{2}{*}{ Sackick, 2003 [12] } & $<1$ & 1.5 & $400-600$ & $30-50$ & 1 \\
\hline & 3-Jan & 3 & $250-370$ & $50-60$ & 1 \\
\hline Dunn, 2004 [7] & $<4$ & 3 & 260 & 55 & $1-2$ \\
\hline
\end{tabular}

\section{Objective}

The purpose of the current pilot study is to report our experience of the most appropriate parameters as well as the effectiveness of the Sciton Profile Nd-Yag laser in the treatment of cutaneous vessels of 0.1 - and 4.0-mm's diameter.

\section{Materials and Methods}

19 patients aged 20-65 yrs., Fitzpatrick types II to IV were treated with Sciton Nd: YAG $1064 \mathrm{~nm}$ laser for red to purple colored vessels and telangiectasia to reticular patterns between 0.1 and $4.0 \mathrm{~mm}$ in diameter. A hand held cooling devise was set to $5 \mathrm{C}$, with topical ultrasound gel, which was applied for a minimum of 3 seconds prior to lasering as well as during and after treatment, Exclusion criteria included pregnancy, vessel varicosities $>4 \mathrm{~mm}$ 's, tendency to keloid formation and infection in the treated area and Fitzpatrick type V. The location of vessels was specified. Patients were treated with a $3 \mathrm{~mm}$ spot size hand-piece. The fluence was set based on the vessel depth and color to be between 120 and $350 \mathrm{~J} /$ $\mathrm{cm}$. The pulse duration was set between 10 and $50 \mathrm{~ms}$. The highest fluence and shortest pulse width which could be tolerated without complication were noted for a specific vessel size and color. We used a hand-held micrometer caliper to evaluate vessel size. Patients were treated one to three consecutive passes with a pulse interval of at least 1 second. No topical anesthesia was applied.

Standardized photographs were taken before, 1 and 2 months and as long as 3 months after treatment using a Lumex-Panasonic macro-digital camera with standardized lighting. Response to treatment was rated on a quartile system: 0 (no clearing), 1 (1\%$25 \%$ clearing), 2 (26\%-50\% clearing), 3 (51\%-75\% clearing), and 4 (76\%-100\% clearing). The average number of passes was 2 . We defined a significant improvement at the time of the treatment was greater than or equal to a $50 \%$ clearing. Side effects were documented and subsequently rated as: none,1 (mild), 2 (moderate), 3 (severe). Response to treatment was assessed by two treating physicians and one Registered Laser Nurse.

\section{Results}

19 pts received 27 treatments, with intervals of at least 6 weeks, the most common laser site was the lower leg. No compressive garments were applied after treatment, no topical anesthetic or antibacterial agents were applied before or after treatment. Significant vessel clearance was seen in over $80 \%$ of patients at 3 month follow-up. The best response for $1 \mathrm{~mm}$ vessels was noted using a short pulse $20 \mathrm{~ms}$ or less; with a fluence of greater than 
$150 \mathrm{~J} / \mathrm{cm}^{2}$ (see Figure 1). Reticular vessels required a longer pulse width: $30-50 \mathrm{~ms}$ and a fluence of $150 \mathrm{~J} / \mathrm{cm}^{2}$ (see Figure 2). Pain was the limiting factor when using shorter pulse widths or higher fluences. Treatment of vessels $4 \mathrm{~mm}$ or greater was limited by the sensation of pain. A lower fluence and longer pulse was associated with a reduction of absence of pain, but without appreciable vessel clearance. Following treatment, no pigmentary changes were noted. There were no reported burns. Matting was not seen, and the commonest complaint was the sensation of heat at higher fluences and short pulse widths. Firm contact cooling with jel was very important in reducing this heat sensation and preventing the complication of burns or pigmentary changes.

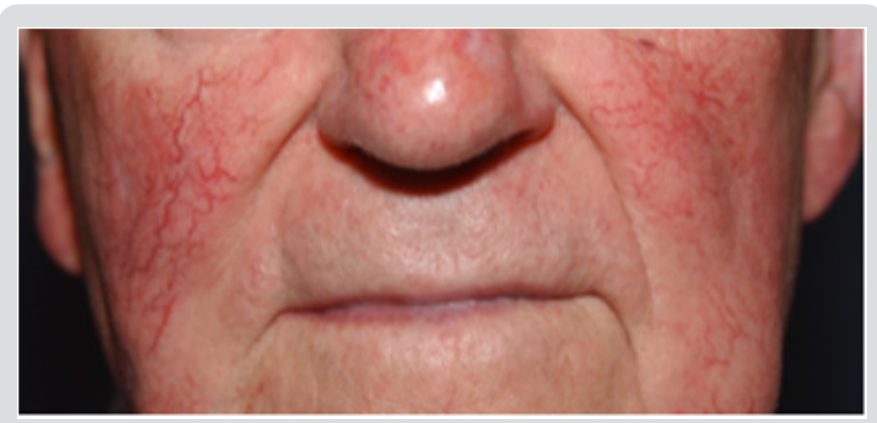

Figure 1: Photograph of $<1 \mathrm{~mm}$ red colored vessels to bilateral cheeks. Treatment only to the left lower cheek: 270 Joules, 15 milliseconds, 3 passes and 2 treatments at 6 week intervals. Photo taken 3 months following last treatment shows $75 \%$ clearing of the vessels.

\section{Discussion}

The current report indicates that the Sciton profile Nd-Yag is a useful laser modality in the reduction of cutaneous vessels of less than $4 \mathrm{~mm}$ in size. Our experience was consistent with other reports [12] concerning Nd-Yag parameters, a shorter pulse duration (15 milliseconds) with higher fluence $\left(>150 \mathrm{~J} / \mathrm{cm}^{2}\right)$ was effective in closing smaller vessels $(<1 \mathrm{~mm})$. Reticular vessels responded well to a longer pulse duration (30-50 milliseconds) with a fluence of $150 \mathrm{~J} / \mathrm{cm}^{2}$. Because of the relatively long wavelength, and continuous skin cooling there were no injuries to the skin and no changes in pigmentation as perceived by either patient or our group. Patient

Table 2: Current recommendations for Nd-YAG settings to reduce cutaneous vessels.

\begin{tabular}{|c|c|c|c|c|c|c|}
\hline \multicolumn{5}{|c|}{ Recommended vascular settings } & \multicolumn{2}{|c|}{ Maximal power output } \\
\hline Laser & vessel size(mm) & spot $\operatorname{size}(\mathrm{mm})$ & pulse duration(msec) & fluence $\left(\mathrm{J} / \mathrm{cm}^{2}\right)$ & pulse duration(msec) & fluence $\left(\mathrm{J} / \mathrm{cm}^{2}\right)$ \\
\hline \multirow{8}{*}{$\begin{array}{c}\text { Laserscope } \\
\text { (LyraXP) }\end{array}$} & $0.5-1.0 \mathrm{leg}$ & 1.5 & $30-50$ & $400-700$ & $20-100$ & 900 \\
\hline & $0.5-1.0$ face $/$ red & 1.5 & $11-16$ & $300-400$ & & \\
\hline & $0.5-1.0$ face/ blue & 1.5 & $11-16$ & $240-340$ & & \\
\hline & Venous lakes: lip & 4.6 & 50 & $80-100$ & & \\
\hline & $1.0-2.0 \mathrm{leg}$ & 3.0 & $50-60$ & $250-370$ & & \\
\hline & 1.0-2.0 port-wine & $50-60$ & & $250-370$ & & \\
\hline & $2.0-4.0$ & 5.0 & $25-30$ & 125 & & \\
\hline & & 10 & $20-100$ & 100 & $20-100$ & 100 \\
\hline
\end{tabular}




\begin{tabular}{|c|c|c|c|c|c|c|}
\hline \multirow{3}{*}{ Cutera (Altus) } & $<0.5$ & 3 & $15-30$ & & $0.1-300$ & 300 \\
\hline & $05 .-1.5$ & $5-7$ & $25-50$ & & $0.1-300$ & \\
\hline & $1-3.5$ & 10 & $40-60$ & & $0.1-300$ & \\
\hline \multirow{4}{*}{ Sciton (Profile) } & $0.1-1.0$ & 3.0 & $5-15$ & $250-350$ & $0.1-60$ & 460 \\
\hline & $1-3$ & 3.0 & $10-25$ & $200-300$ & $0.1-60$ & \\
\hline & $3-4$ & 3.0 & $15-20$ & $200-300$ & & \\
\hline & & 6.0 & 150 & & & \\
\hline \multirow{4}{*}{ Candela (VP-YAG) } & $<1.5$ & 1.5 & 20 & $250-290$ & $0.25-300$ & 460 \\
\hline & $1-2$ & 3 & 30 & $220-250$ & & \\
\hline & $3-4$ & 6 & $?$ & $<200$ & $0.25-300$ & 200 \\
\hline & & 10 & & & $0.25-300$ & 100 \\
\hline
\end{tabular}

Table 3: Current recommendations for Nd-YAG settings to reduce cutaneous vessels.

\begin{tabular}{|c|c|c|c|c|c|c|}
\hline \multicolumn{5}{|c|}{ Recommended vascular settings } & \multicolumn{2}{|c|}{ Maximal power output } \\
\hline L Laser & vessel size $(\mathrm{mm})$ & $\operatorname{spot} \operatorname{size}(\mathrm{mm})$ & $\begin{array}{c}\text { pulse } \\
\text { duration(msec) }\end{array}$ & fluence $\left(\mathrm{J} / \mathrm{cm}^{2}\right)$ & pulse duration(msec) & fluence $\left(\mathrm{J} / \mathrm{cm}^{2}\right)$ \\
\hline \multirow{8}{*}{$\begin{array}{l}\text { Laserscope } \\
\text { (LyraXP) }\end{array}$} & $0.5-1.0 \mathrm{leg}$ & 1.5 & $30-50$ & $400-700$ & $20-100$ & 900 \\
\hline & $0.5-1.0$ face /red & 1.5 & $11-16$ & $300-400$ & & \\
\hline & $0.5-1.0$ face/ blue & 1.5 & $11-16$ & $240-340$ & & \\
\hline & Venous lakes: lip & 4.6 & 50 & $80-100$ & & \\
\hline & $1.0-2.0 \mathrm{leg}$ & 3.0 & $50-60$ & $250-370$ & & \\
\hline & $1.0-2.0$ port-wine 3.0 & 3.0 & $50-60$ & $250-370$ & & \\
\hline & $2.0-4.0$ & 5.0 & $25-30$ & 125 & & \\
\hline & & 10 & $20-100$ & 100 & $20-100$ & 100 \\
\hline \multirow{4}{*}{$\begin{array}{c}\text { Cutera (Altus ) } \\
\text { (Coolglide Excel) }\end{array}$} & $<0.5$ & 3 & $15-30$ & & $0.1-300$ & 300 \\
\hline & $0.5-1.5$ & $5-7$ & $25-50$ & & $0.1-300$ & 300 \\
\hline & $1-3.5$ & $5-7$ & $25-50$ & & $0.1-300$ & \\
\hline & & & & & $0.1-300$ & \\
\hline \multirow{4}{*}{ Sciton (Profile) } & $0.1-1.0$ & 3.0 & $5-15$ & $250-350$ & $0.1-60$ & 460 \\
\hline & $1-3$ & 3.0 & $10-25$ & $200-300$ & $0.1-60$ & \\
\hline & $3-4$ & 3.0 & $15-20$ & $200-300$ & & \\
\hline & & 6.0 & 150 & & & \\
\hline \multirow{4}{*}{ Candela (VP-YAG) } & $<1.5$ & 1.5 & 20 & $250-290$ & $0.25-300$ & 460 \\
\hline & $1-2$ & 3 & 30 & $220-250$ & & \\
\hline & $3-4$ & 6 & $?$ & $<200$ & $0.25-300$ & 200 \\
\hline & & 10 & ? & & $0.25-300$ & 100 \\
\hline
\end{tabular}

\section{References}

1. Lupton JR, Alster TS, Romero P (2002) Clinical comparison of sclerotherapy versus long-pulsed Nd:YAG laser treatment for lower extremity telangiectases. Dermatol Surg 28(8): 694-697.
2. Samson RH (2004) Spider vein treatment: Sclerotherapy vs photoderm therapy - a clinical analysis of results. International Vein Conference: In office techniques, Key Biscayne, Fl.

3. Rogachefsky AS, Silapunt S, Goldberg DJ (2002) Nd:YAG laser (1064 nm) irradiation for lower extremity telangiectases and small reticular veins: 
efficacy as measured by vessel color and size. Dermatol Surg 28(3): 220223.

4. Troxler M (2000) One clinic's experience in the treatment of varicose veins and leg telangiecastasias with the VascuLight tm Intense Pulse Light/Nd: YAG Laser source. Clin Applic Notes 8(3): 1-4.

5. Levy JL, Elbahr C, Jouve E, Mordon S (2004) Comparison and sequential study of long pulsed Nd:YAG 1,064 nm laser and sclerotherapy in leg telangiectasias treatment. Lasers Surg Med 34(3): 273-276.

6. Bush RG (2004) Combination sclerotherapy / $940 \mathrm{~nm}$ diode laser to treat telangiectasias. International Vein Conference: In office techniques, Key Biscayne, Fl.

7. Dunne JV (2004) Live case Semonstration: Laser therapy of telangiectasias: 1064 Neo:YAG lasers. International Vein Conference: In office techniques, Key Biscayne, Fl.

8. Min RJ, Zimmet SE, Isaacs MN, Forrrestal MD (2001) Endovenous laser treatment of the incompetent greater saphenous vein. JVIR 12(10): $1167-1171$

9. Chandler JG, Pichot O, Seesa C, Schuller Petrovic S, Kabnick LS, et al. (2000) Treatment of primary venous insufficiency by endovenous saphenous vein obliteration. Vasc Surg 34: 201-214.

10. Kabnick LS (2004) Phlebectomy is better than ultra-sound guided foam sclerotherapy to clean up varicose veins after saphenous ablation. International Vein Conference: In office techniques, Key Biscayne, Fl.

11. Kahan RS (2003) Letter to the editor. Laser Surg Med 32: 251.

12. Sadick NS (2003) Laser treatment with a 1064-nm laser for lower extremity Class I-III veins employing variable spots and pulse width parameters. Dermatol Surg 29(9): 916-919.

13. Wheeland RG (2004) Laser, light - based treatments multiply, satisfy need for less invasive therapies. Cosmet Surg Times pp. 17-19.

ISSN: 2574-1241

DOI: 10.26717/BJSTR.2019.19.003365

Rex Moulton Barrett. Biomed J Sci \& Tech Res

(c) (P) This work is licensed under Creative

Submission Link: https://biomedres.us/submit-manuscript.php
14. Moretti M (2004) Vascular comparison chart. Aesthetic Buyers Guide $7(1): 72-74$.

15. Eremia S, Li C, Umar SH (2002) A side-by-side comparative study of 1064 $\mathrm{nm}$ Nd:YAG, $810 \mathrm{~nm}$ diode and $755 \mathrm{~nm}$ alexandrite lasers for treatment of 0.3-3 mm leg veins. Dermatol Surg 28(3): 224-230.

16. Massey RA, Katz BE (1999) Successful treatment of spider leg veins with a high - energy, long-pulse, frequency - doubled Neodynium: YAG Laser (Help-G). Dermatol Surg 25(9): 677-680.

17. Weiss RA, Weiss MA (1999) Early clinical results with a multiple synchronized pulse $1064 \mathrm{NM}$ laser for leg telangiectasias and reticular veins. Dermatol Surg 25(5): 399-402.

18. Colaiuda S, Colaiuda F, Gasparotti M (2000) Treatment of deep underlying reticular veins by Nd: Yag laser and IPL source. Minerva Cardioangiol 48(10): 329-334.

19. Wilder D (1999) Pulsed 1064-nm Nd: YAG Laser Therapy for Noninvasive Treatment of a Massive Hemangioma: Case Report. J Clin Laser Med \& Surg 17(6): 245-247.

20. Major A, Brazzini B, Campolmi P, Bonan P, Mavilia L, et al. (2001) Nd:YAG $1064 \mathrm{~nm}$ laser in the treatment of facial and leg telangiectasias. J Eur Acad Dermatol Venereol 15(6): 559-565.

21. Rothaus K Evaluation of the Lyra Nd:YAG $1064 \mathrm{~nm}$ wavelength for the treatment of leg vessels up to $4 \mathrm{~mm}$ in diameter. Laserscope.

22. Omura NE, Dover JS, Arndt KA, Kauvar AN (2003) Treatment of reticular leg veins with a $1064 \mathrm{~nm}$ long-pulsed Nd:YAG laser. J Am Acad Dermatol 48(1): 76-81.

23. Sadick NS (2001) Long-term results with a multiple synchronizedpulse $1064 \mathrm{~nm} \mathrm{Nd:YAG} \mathrm{laser} \mathrm{for} \mathrm{the} \mathrm{treatment} \mathrm{of} \mathrm{leg} \mathrm{venulectasias} \mathrm{and}$ reticular veins. Dermatol Surg 27(4): 365-369.

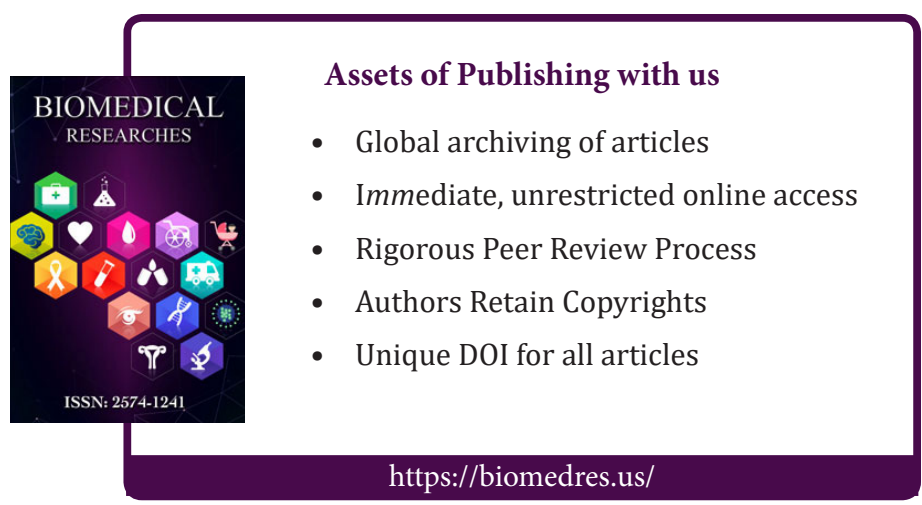

